\title{
Simultaneous Superficial Temporal Artery to Middle Cerebral or Anterior Cerebral Artery Bypass With Pan-synangiosis for Moyamoya Disease Covering Both Anterior and Middle Cerebral Artery Territories
}

\author{
-Technical Note-
}

\author{
Tatsuya ISHIKAWA, Hiroyasu KAMIYAMA*, Satoshi KURODA, \\ Hiroshi YASUDA**, Naoki NAKAYAMA, and Katsumi TAKIZAWA* \\ Department of Neurosurgery, Hokkaido University Graduate School of Medicine, Sapporo, \\ Hokkaido; * Department of Neurosurgery, Asahikawa Red-Cross Hospital, Asahikawa, \\ Hokkaido; ** Sapporo Azabu Neurosurgical Hospital, Sapporo, Hokkaido
}

\begin{abstract}
Some patients with moyamoya disease treated by conventional surgical procedures may develop postoperative refractory ischemia and perioperative cerebral infarction in the anterior cerebral artery (ACA) territory. We present a novel operative procedure for moyamoya disease to avoid the risk of ischemia in the ACA territory, which consists of simultaneous superficial temporal artery (STA) to middle cerebral artery (MCA) or ACA bypass with pan-synangiosis, encephalo-duro-arterio-myosynangiosis for the lateral frontal and temporal areas, and encephalo-galeo-arterio-synangiosis for the medial frontal area. This procedure can establish direct bypass to the ACA territory at the first intervention. Simultaneous STA-MCA and STA-ACA bypasses with pan-synangiosis is suitable for patients with moyamoya disease associated with severely impaired perfusion of the ACA territory requiring direct bypass surgery.
\end{abstract}

Key words: moyamoya disease, superficial temporal artery-anterior cerebral artery bypass, encephalo-galeo-arterio-synangiosis, anterior cerebral artery, intellectual outcome

\section{Introduction}

Moyamoya disease, an occlusive disease of the circle of Willis, is characterized by the progressive stenosis of the bilateral internal carotid arteries at the terminal portion and the appearance of networklike moyamoya vessels at the base of the brain. Various methods of vasoreconstructive surgery are accepted as treatment for moyamoya disease, including direct bypass such as superficial temporal artery (STA) to middle cerebral artery (MCA) bypass ${ }^{8)}$; indirect bypass such as encephalo-duroarterio-synangiosis, ${ }^{20)}$ encephalo-duro-arterio-myosynangiosis (EDAMS), ${ }^{12)}$ and omentum transplantation $^{9)}$; multiple burr holes ${ }^{1,11)}$; and a combination of direct and indirect bypasses.,10,12) Such methods provide good neovascularization through the extracranial arteries and reduced risk of cerebral ischemia after the surgery. Revascularization surgery for pediatric moyamoya disease has been consistently effective in eliminating ischemic neurological symptoms irrespective of the use of direct or indirect bypass surgery. ${ }^{6,8,12,19)}$

Vasoreconstructive surgery is usually performed to cover the lateral frontal, temporal, and parietal lobes, which are territories of the MCA, with satisfactory results in most patients. However, patients have suffered refractory ischemic episodes due to insufficient flow to the anterior cerebral artery (ACA) territory. ${ }^{5,7,17)}$ Vascular reconstruction targeted to the territory of the ACA is important, because insufficient blood flow in the ACA territory

Received October 13, 2005; Accepted May 10, 2006

Author's present address: T. Ishikawa, M.D., Department of Neurosurgery, Saitama Medical Center, Kawagoe, Saitama, Japan. 
Table 1 Profiles of the patients undergoing simultaneous superficial temporal artery to middle cerebral or anterior cerebral artery bypass with pan-synangiosis

\begin{tabular}{|c|c|c|c|c|c|c|c|c|}
\hline $\begin{array}{l}\text { Case } \\
\text { No. }\end{array}$ & $\begin{array}{l}\text { Preoperative } \\
\text { symptoms }\end{array}$ & $\begin{array}{l}\text { Age at } \\
\text { onset } \\
\text { (yrs) }\end{array}$ & $\begin{array}{l}\text { Age at } \\
\text { surgery } \\
\text { (yrs) }\end{array}$ & Sex & $\begin{array}{l}\text { First } \\
\text { surgery } \\
\text { side }\end{array}$ & $\begin{array}{l}\text { Second } \\
\text { surgery } \\
\text { side }\end{array}$ & $\begin{array}{l}\text { Perioperative } \\
\text { complications }\end{array}$ & Postoperative symptoms \\
\hline 1 & TIA & 7 & 9 & $\mathrm{~F}$ & $\mathrm{rt}$ & lt & none & TIA disappeared \\
\hline 2 & TIA & 10 & 12 & $\mathrm{~F}$ & lt & rt & none & TIA disappeared \\
\hline 3 & TIA & 3 & 5 & $\mathrm{~F}$ & lt & $\mathrm{rt}$ & none & TIA disappeared \\
\hline 4 & TIA & 5 & 6 & $\mathrm{~F}$ & $\mathrm{rt}$ & lt & none & TIA disappeared \\
\hline 5 & headache & 6 & 8 & $\mathrm{M}$ & lt & $\mathrm{rt}$ & none & headache attenuated \\
\hline 6 & TIA & 7 & 10 & M & lt & $\mathrm{rt}$ & $\begin{array}{l}\text { tiny cerebral } \\
\text { infarct }\end{array}$ & TIA frequency reduced \\
\hline 7 & infarct & 3 & 6 & $\mathrm{~F}$ & $\mathrm{rt}$ & & none & $\begin{array}{l}\text { no more ischemic episodes, } \\
\text { improved intellectual ability } \\
\text { (Tanaka-Binet Intelligence } \\
\text { Scale: } 56 \rightarrow 76 \text { ) }\end{array}$ \\
\hline 8 & $\begin{array}{l}\text { TIA, } \\
\text { headache }\end{array}$ & 5 & 14 & $\mathrm{~F}$ & lt & $\mathrm{rt}$ & none & TIA disappeared \\
\hline 9 & $\begin{array}{l}\text { infarct, } \\
\text { seizure }\end{array}$ & 25 & 25 & $\mathrm{~F}$ & lt & $\mathrm{rt}$ & none & $\begin{array}{l}\text { no more ischemic episodes, } \\
\text { improved intellectual ability } \\
\text { (WAIS-R: } 66 \rightarrow 81 \text { ) }\end{array}$ \\
\hline 10 & infarct & 30 & 31 & $\mathrm{~F}$ & lt & & none & $\begin{array}{l}\text { no more ischemic episodes, } \\
\text { improved intellectual ability } \\
\text { (WAIS-R: } 72 \rightarrow 89 \text { ) }\end{array}$ \\
\hline 11 & infarct & 23 & 23 & $\mathrm{M}$ & lt & & none & no more ischemic episodes \\
\hline 12 & TIA & 25 & 25 & $\mathrm{~F}$ & $\mathrm{rt}$ & lt & none & TIA disappeared \\
\hline 13 & TIA & 32 & 32 & $\mathrm{~F}$ & lt & & none & TIA frequency reduced \\
\hline 14 & TIA & 59 & 59 & M & lt & & none & TIA disappeared \\
\hline 15 & TIA & 38 & 38 & $\mathrm{~F}$ & rt & lt & none & TIA disappeared \\
\hline 16 & TIA & 47 & 47 & $\mathrm{~F}$ & rt & & none & TIA disappeared \\
\hline
\end{tabular}

TIA: transient ischemic attack, WAIS-R: Wechsler Adult Intelligence Scale-Revised.

would result in cerebral infarction before reoperative surgery. ${ }^{15)}$ However, such reconstruction is based on indirect bypass, which has no immediate effect to improve cerebral perfusion, and is sometimes unsuccessful especially in adult patients with moyamoya disease. Revascularization from indirect bypass by encephalo-myo-synangiosis ${ }^{8)}$ or EDAMS ${ }^{12)}$ begins to develop 2 weeks after surgery and becomes well developed at 3 months postoperatively.

Here we describe a method of simultaneous STAMCA and STA-ACA bypass with pan-synangiosis to establish both direct and indirect bypasses to both ACA and MCA territories, thus providing an effective vasoreconstructive procedure for moyamoya disease associated with impaired blood flow in the ACA territory.

\section{Materials and Methods}

We have performed vasoreconstructive surgery in 35 hemispheres of 22 patients with moyamoya disease since 1996. During this period, 26 hemispheres of 16 patients, four males and 12 females aged 5 to 59 years, were treated with simultaneous STA-MCA and STA-ACA bypass with pan-synangiosis (EDAMS for the lateral frontal and temporal areas and encephalo-galeo-arterio-synangiosis [EGAS] for the medial frontal area). Selection criteria for STA-ACA and STA-MCA bypasses were based on cerebral blood flow (CBF) study and angiographical findings; i.e. impaired blood flow in the ACA territory, and occlusion of the proximal portion of the ACA. STA-ACA bypass was not performed if no branches of the ACA were suitable for anastomosis. Clinical profiles of the patients who underwent the complete procedure including STA-ACA bypass are shown in Table 1. Moyamoya disease manifested as transient ischemic attacks (TIAs) in 11 patients, cerebral infarction in four, and only headache in one.

\section{Surgical Procedures}

The surgical technique for the lateral area of the brain was previously reported. ${ }^{4)}$ This technique can establish both direct and indirect bypasses even in 
the medial frontal area. The skin incision is made along the course of the parietal branch of the STA and extended upward to the midline near the bregma and then along the midline downward to the hairline (Fig. 1A). Using this larger skin flap, a longer portion of the frontal branch of the STA can be dissected which is adequate to reach the medial frontal area. The temporal muscle is separated as widely as possible. The galea of the frontal skin flap is also dissected, preserving a small vascular pedicle from the frontal branch of the STA (Fig. 1B). The normal frontotemporal craniotomy is performed, which can be covered with the temporal muscle flap, preserving the middle meningeal artery. The medial frontal craniotomy, which should be matched to the size of the galeal flap, is made separately (Fig. 1C). A larger craniotomy might be lead to less favorable cosmetic results. These two separate craniotomies preserve a bridge along the linear temporalis, and thus can retain the original shape of the skull.

The dura is then opened to preserve the main branch of the middle meningeal artery. Special attention is needed to avoid injury to the frontal cortical veins draining into the superior sagittal sinus. Selection of the branch of the ACA to act as recipient of the STA-ACA bypass is sometimes difficult. The area under the medial frontal craniotomy is the border zone between the ACA and MCA territories. We select a branch of the ACA passing upward from the interhemispheric fissure as the recipient. The frontal branch of the STA is then dissected very carefully to reach the recipient artery. The STA-ACA anastomosis is performed in an end-to-side fashion (Fig. 1D). STA-ACA bypass is technically more difficult than STA-MCA bypass, because the diameters of the anastomosed vessels are smaller. One or two STA-MCA bypasses are also established using other branches of the STA (Fig. 1D).

EDAMS is then performed in the usual way to cover the lateral craniotomy, and EGAS to cover the dural opening of the medial frontal craniotomy with the vascularized galeal flap (Fig. 1E). Cranioplasty is then performed for both craniotomies using bone flaps. Bone gaps are filled with bone chips and fibrin glue to avoid postoperative depression of the skull and face.

\section{Results}

One pediatric patient suffered postoperative cerebral infarction in the frontal lobe manifesting as transient restless consciousness. Minor skin problems (localized alopecia along the skin incision) occurred although the STA branches were exten-

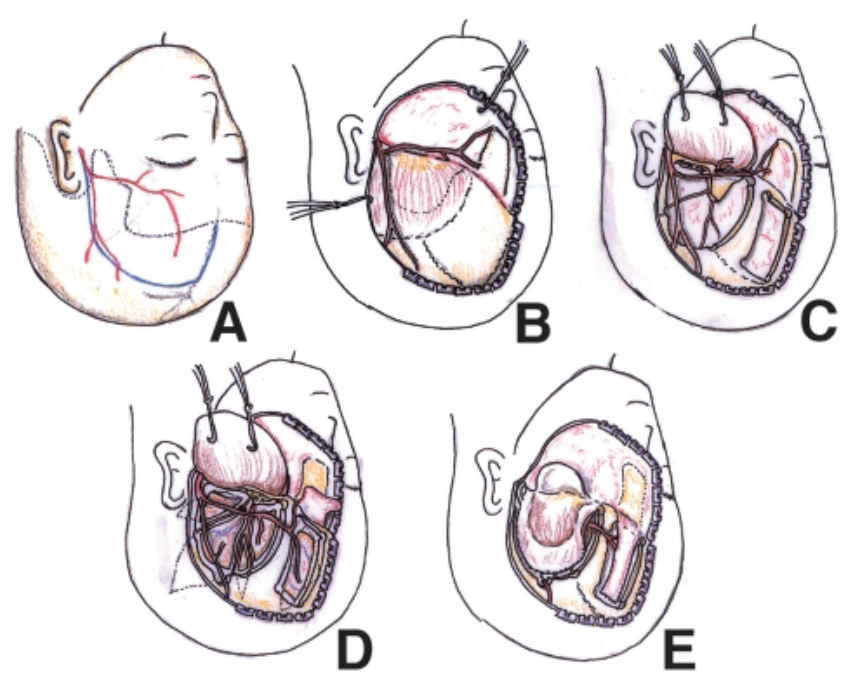

Fig. 1 Schematic drawings of the operative procedures. Skin incision is made along the course of the parietal branch of the superficial temporal artery (STA) and extended upward to the midline near the bregma and then along the midline downward to the hairline (A). Using this large skin flap, an adequate portion of the frontal branch of the STA can be dissected to reach the medial frontal area. The temporal muscle is separated as widely as possible. The galea of the frontal skin flap is also dissected, preserving a small vascular pedicle from the frontal branch of the STA (B). Two separate craniotomies are made, a frontotemporal craniotomy, which can be covered with the temporal muscle flap, and a medial frontal craniotomy, which can be covered with the galeal flap (C). The STA-anterior cerebral artery bypass and the STA-middle cerebral artery bypass are established (D). Encephalo-duro-arterio-myo-synangiosis is performed over the lateral craniotomy, and encephalo-galeo-arterio-synangiosis over the dural opening of the medial frontal craniotomy using the vascularized galeal flap (E).

sively separated from the scalp.

TIA completely disappeared in nine of the 11 patients presenting with TIA, and the frequency of TIA markedly reduced in the other two. Symptoms improved in all four patients presenting with cerebral infarction. Intellectual ability apparently improved in three of the four patients. Headache was attenuated in one of the two patients presenting with headache. 


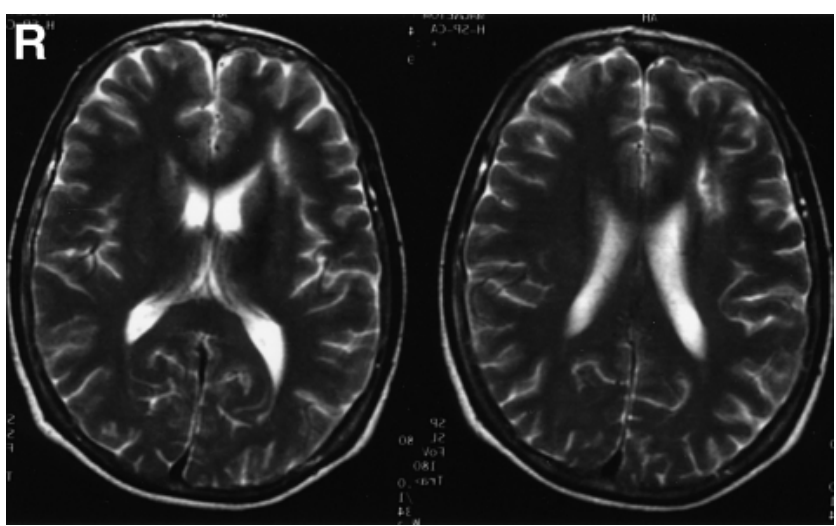

Fig. 2 Representative case of a 25-year-old woman (Case 9). $T_{2}$-weighted magnetic resonance images showing cerebral infarction in the subcortical area of the left frontal lobe.
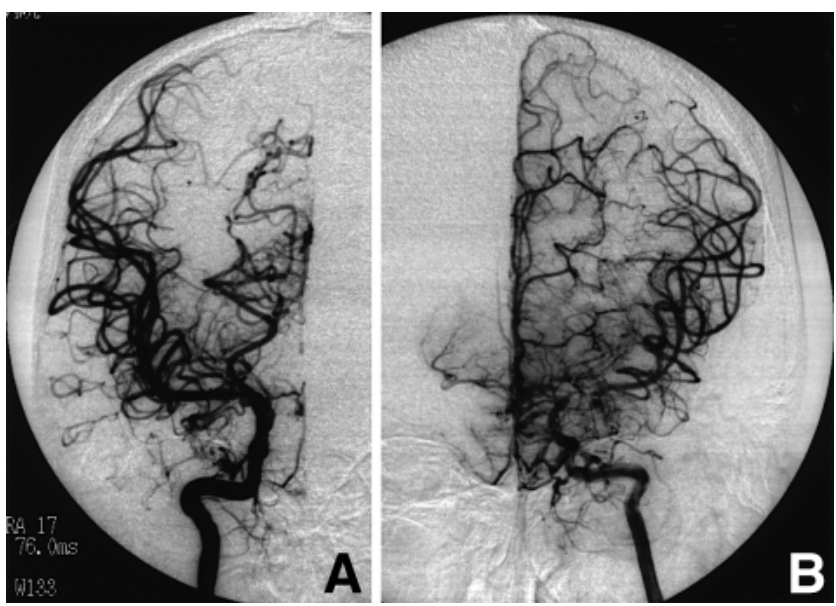

Fig. 3 Case 9. Right (A) and left (B) carotid angiograms, anteroposterior views, showing severe stenosis of the left internal carotid artery at the terminal portion associated with dilated moyamoya vessels, and mild stenosis of the right internal carotid artery and occlusion of the right anterior cerebral artery at the $A_{1}$ segment.

\section{Illustrative Case Report}

A 25-year-old woman (Case 9) visited our hospital complaining of dullness in the right upper extremity, emotional changes, and speech disturbance. Neurological examination found slight right hemiparesis and motor dominant aphasia. Magnetic resonance imaging showed cerebral infarction in the subcortical area of the left frontal lobe (Fig. 2). Cerebral angiography revealed severe stenosis of the left internal carotid artery at the terminal portion associated with dilated moyamoya vessels, and mild
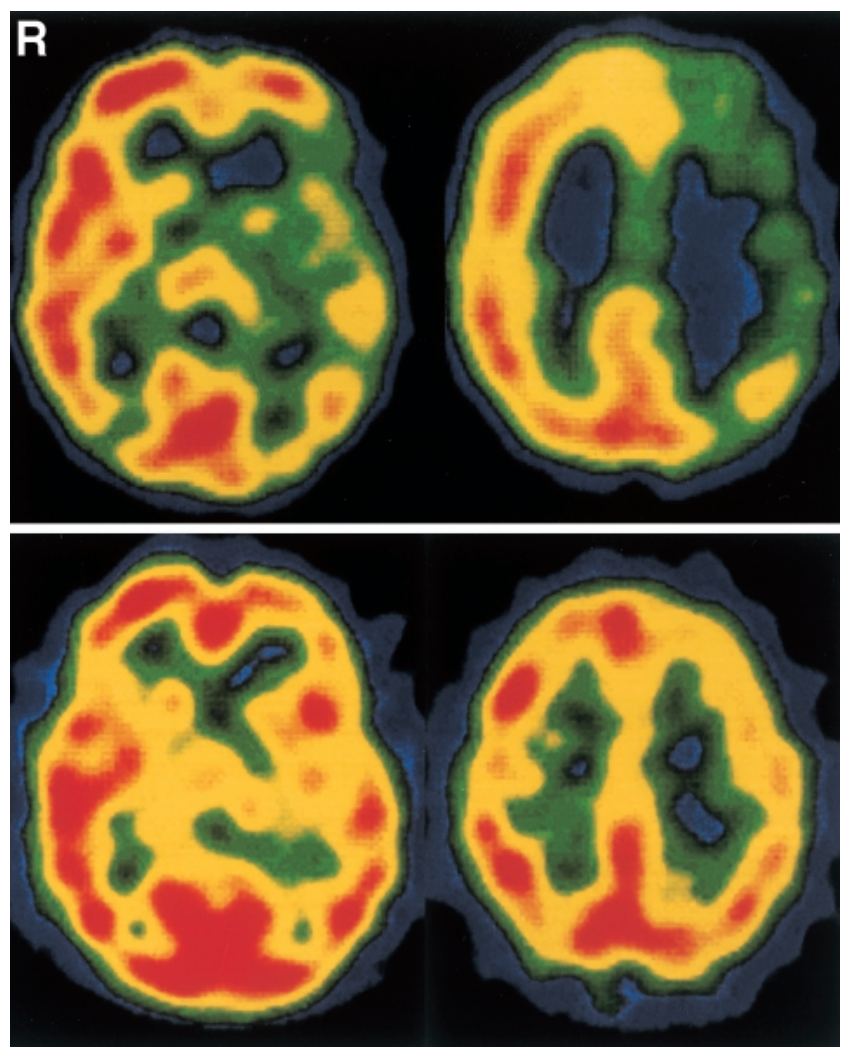

Fig. 4 Case 9. Preoperative (upper row) and postoperative (lower row) single photon emission computed tomography scans showing preoperative severe attenuation of the regional cerebral blood flow (rCBF) in both left anterior cerebral artery (ACA) and middle cerebral artery (MCA) territories, and postoperative markedly improved $\mathrm{rCBF}$ in both ACA and MCA territories.

stenosis of the right internal carotid artery and occlusion of the right ACA at the $A_{1}$ segment (Fig. 3). Single photon emission computed tomography (SPECT) showed severe attenuation of the regional CBF (rCBF) in both left ACA and MCA territories (Fig. 4 upper row).

She first underwent direct and indirect bypass surgery on the left. The proximal portion of the right ACA was occluded, so she also underwent the same procedure on the right 2 weeks after the first surgery. Postoperative course was uneventful. She completely recovered from the hemiparesis and aphasia. Postoperative left external carotid angiography 3 months after the surgery showed neovascularization from the direct and indirect bypasses (Fig. 5A). The stenosis of the right ACA was severe, but the STA-ACA bypass was patent and the STA was enlarged (Fig. 5B). The stenosis of the MCA was not so severe, so neovascularization in the 


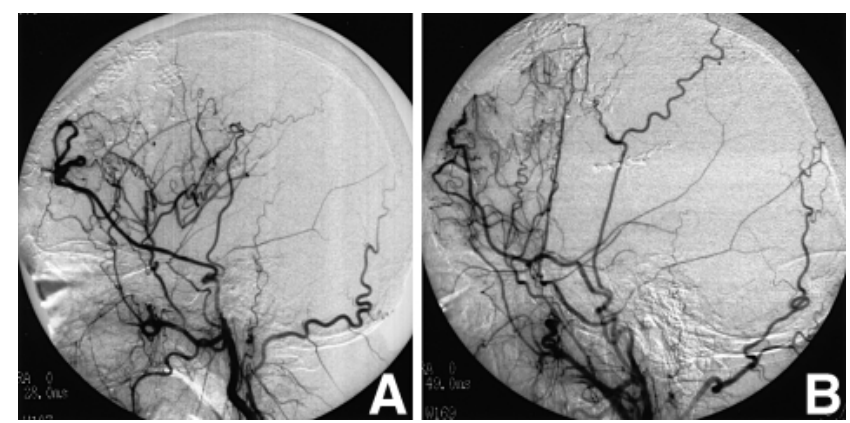

Fig. 5 Case 9. Left (A) and right (B) carotid angiograms, lateral views, taken at 3 months after vasoreconstructive surgery showing neovascularization from the direct and indirect bypasses on the left, and the patent superficial temporal artery (STA)-anterior cerebral artery bypass and enlarged STA on the right, but with less neovascularization in the middle cerebral artery territory.

MCA territory was not so rich at this time (Fig. 5B). Postoperative SPECT showed improved rCBF including the medial frontal area (Fig. 4 lower row). Her intellectual ability on the Wechsler Adult Intelligence Scale-Revised had increased from 66 in the preoperative period to 81 in the postoperative period. She returned to her previous job 4 months after the onset.

\section{Discussion}

TIAs in the lower extremities are known to persist after vasoreconstructive surgery in some patients. In particular, repetitive paraparetic TIA after the first operation (STA-MCA bypass and encephalomyo-synangiosis) was associated with poor cerebral hemodynamics in the frontal lobe in eight of 25 patients with pediatric moyamoya disease. ${ }^{22)}$ Various vasoreconstructive procedures for the frontal lobe have been performed. Direct STA-ACA bypass surgery was performed as a secondary surgery for refractory ischemic episodes. ${ }^{5,7)}$ Indirect bypass surgery targeting the frontal lobe, ${ }^{21)}$ ribbon EDAMS,13) burr holes, ${ }^{1,11)}$ and dural pedicle insertion over the brain surface combined with encephalo-galeo-synangiosis ${ }^{23}$ have also been reported. The present study describes the technical procedure to establish vasoreconstructive surgery including direct bypass for the ACA territory at the first intervention.

Known risk factors for perioperative ischemic complications include frequent TIAs, indirect bypass procedures, hemodynamic compromise, dehydration, lacrimation, hypotension, and hyper- capnia as well as hypocapnia. ${ }^{14)}$ Blood flow directly supplied by the STA-ACA bypass, even if only a very small amount, may be helpful in preventing the perioperative risk of stroke and terminating postoperative ischemic symptoms, as found with STA-MCA bypass. ${ }^{6}$ Indeed, we treated two patients who suffered infarction in the ACA territory after STA-MCA bypass combined with EDAMS. ${ }^{15)}$ We speculate that the blood supply to the ACA territory in those patients may have worsened with reduction of collateral flow through the moyamoya vessels as a result of changes in the hemodynamics after the STA-MCA bypass combined with EDAMS. ${ }^{14)}$ Avoiding the risk of hypoperfusion in the ACA territory is also important in the perioperative period for improving the outcome, especially if the patient has impaired preoperative hemodynamics in the ACA territory. On the other hand, if the ischemia is located only in the MCA territory, we should anastomose pedicles of the STA mainly to branches of the MCA.

Vasoreconstructive surgery through "small craniotomy" does not improve cerebral hemodynamics in the frontal lobe. ${ }^{14,18,22)}$ Intellectual outcome is an important consideration in treating patients with moyamoya disease. ${ }^{10)}$ Recent studies have suggested a close relationship between intellectual development and frontal lobe function in children. ${ }^{2)}$ Chronic blood flow reduction in the frontal lobe may be responsible for poor intellectual outcome. Ischemic attacks were still observed after a mean of 6 years 11 months in half of a series of the TIA-type patients treated nonsurgically, whereas the ischemic symptoms disappeared within 5 years after onset in the other half. ${ }^{3)}$ The TIA-type patients had a good intellectual prognosis. ${ }^{3)}$ Other studies have suggested that intellectual impairment would progress, even in TIA-type patients, if they did not undergo appropriate surgical treatment. ${ }^{16,19)}$ We are not certain whether direct STA-ACA bypass is always necessary or only indirect bypass targeting the medial frontal area such as EGAS is sufficient for this intellectual improvement. Further follow-up studies are necessary.

In conclusion, simultaneous STA-MCA and STAACA bypasses with pan-synangiosis are suitable for patients with moyamoya disease associated with severely impaired perfusion in the ACA territory requiring direct bypass surgery. The surgical procedure may prevent intellectual deterioration and improve the overall outcome by normalizing cerebral hemodynamics in the frontal lobe. 


\section{References}

1) Endo M, Kawano N, Miyasaka Y, Yada K: Cranial burr hole for revascularization in moyamoya disease. J Neurosurg 71: 180-185, 1989

2) Filley CM: Clinical neurology and executive dysfunction. Semin Speech Lang 21: 95-108, 2000

3) Fukuyama Y, Umezu R: Clinical and cerebral angiographic evaluations of idiopathic progressive occlusive disease of the circle of Willis ('moyamoya' disease) in children. Brain Dev 7: 21-37, 1985

4) Houkin K, Kamiyama H, Takahashi A, Kuroda S, Abe $\mathrm{H}$ : Combined revascularization surgery for childhood moyamoya disease: STA-MCA and encephaloduro-arterio-myo-synangiosis. Childs Nerv Syst 13: 24-29, 1997

5) Ishii R, Koike T, Takeuchi S, Ohsugi S, Tanaka R, Konno K: Anastomosis of the superficial temporal artery to the distal anterior cerebral artery with interposed cephalic vein graft. Case report. J Neurosurg 58: 425-429, 1983

6) Ishikawa T, Houkin K, Kamiyama H, Abe H: Effects of surgical revascularization on outcome of patients with pediatric moyamoya disease. Stroke 28: 1170-1173, 1997

7) Iwama T, Hashimoto N, Miyake H, Yonekawa Y: Direct revascularization to the anterior cerebral artery territory in patients with moyamoya disease: report of five cases. Neurosurgery 42: 1157-1162, 1998

8) Karasawa J, Kikuchi H, Furuse S, Kawamura J, Sakai T: Treatment of moyamoya disease with STA-MCA anastomosis. J Neurosurg 49: 679-688, 1978

9) Karasawa J, Kikuchi H, Kawamura J, Sakai T: Intracranial transplantation of the omentum for cerebrovascular moyamoya disease. Surg Neurol 32: 527-531, 1993

10) Karasawa J, Touho H, Ohnishi H, Miyamoto S, Kikuchi H: Long-term follow-up study after extracranial-intracranial bypass surgery for anterior circulation ischemia in childhood moyamoya disease. J Neurosurg 77: 84-89, 1992

11) Kawaguchi T, Fujita S, Hosoda K, Shose Y, Hamano $\mathrm{S}$, Iwakura $\mathrm{M}$, Tamaki $\mathrm{N}$ : Multiple burr-hole operation for adult moyamoya disease. J Neurosurg 84: 468-476, 1996

12) Kinugasa K, Mandai S, Kamata I, Sugiu K, Ohmoto T: Surgical treatment of moyamoya disease: operative technique for encephalo-duro-arterio-myo-synangiosis, its follow-up, clinical results, and angiograms. Neurosurgery 32: 527-531, 1993

13) Kinugasa K, Mandai S, Tokunaga K, Kamata I, Sugiu K, Handa A, Ohmoto T: Ribbon encephalo-duroarterio-myo-synangiosis for moyamoya disease. Surg Neurol 41: 455-461, 1994

14) Kuroda S, Houkin K, Ishikawa T, Nakayama N, Ikeda J, Ishii N, Kamiyama H, Iwasaki Y: Determinants of intellectual outcome after surgical revascularization in pediatric moyamoya disease: a multivariate analysis. Childs Nerv Syst 20: 302-308, 2004

15) Kuroda S, Houkin K, Nunomura M, Abe H: Frontal lobe infarction due to hemodynamic change after surgical revascularization in moyamoya diseasetwo case reports. Neurol Med Chir (Tokyo) 40: 315-320, 2000
16) Kurokawa T, Tomita S, Ueda K, Narazaki O, Hanai T, Hasuo K, Matsushima T, Kitamura K: Prognosis of occlusive disease of the circle of Willis (moyamoya disease) in children. Pediatr Neurol 1: 274-277, 1985

17) Matsushima T, Fujiwara S, Nagata S, Fujii K, Fukui $\mathrm{K}$, Hasuo K: Reoperation for moyamoya disease refractory to encephalo-duro-arterio-synangiosis. Acta Neurochir (Wien) 107: 129-132, 1990

18) Matsushima $T$, Inoue $T$, Suzuki SO, Fujii K, Inoue T, Fukui M, Hasuo K: Surgical treatment of moyamoya disease in pediatric patients - comparison between the results of indirect and direct revascularization techniques. Neurosurgery 31: 401-405, 1992

19) Matsushima $Y$, Aoyagi $M$, Koumo $Y$, Takasato $Y$, Yamaguchi T, Masaoka H, Suzuki R, Ohno K: Effects of encephalo-duro-arterio-synangiosis on childhood moyamoya patients - swift disappearance of ischemic attacks and maintenance of mental capacity. Neurol Med Chir (Tokyo) 31: 708-714, 1991

20) Matsushima Y, Aoyagi M, Masaoka H, Suzuki R, Ohno K: Mental outcome following encephaloduroarteriosynangiosis in children with moyamoya disease with the onset earlier than 5 years of age. Childs Nerv Syst 6: 440-443, 1990

21) Suzuki Y, Negoro M, Shibuya M, Yoshida J, Negoro $\mathrm{T}$, Watanabe K: Surgical treatment for pediatric moyamoya disease: Use of the superficial temporal artery for both areas supplied by the anterior and middle cerebral arteries. Neurosurgery 40: 324-330, 1997

22) Touho H, Karasawa J, Ohnishi H: Preoperative and postoperative evaluation of cerebral perfusion and vasodilatory capacity with 99m Tc-HMPAO SPECT and acetazolamide in childhood Moyamoya disease. Stroke 27: 282-289, 1996

23) Yoshida YK, Shirane R, Yoshimoto T: Non-anastomotic bypass surgery for childhood moyamoya disease using dural pedicle insertion over the brain surface combined with encephalogaleomyosynangiosis. Surg Neurol 51: 404-411, 1999

Address reprint requests to: T. Ishikawa, M.D., Department of Neurosurgery, Saitama Medical Center, 1981 Kamoda, Kawagoe, Saitama 350-8550, Japan.

\section{Commentary}

Ishikawa and colleagues are congratulated on fantastic outcomes in 16 cases where unilateral $(n=6)$ or bilateral $(n=10)$ extensive combined direct and indirect ACA and MCA revascularization was performed for moyamoya disease. The procedure is well described, including low flow (direct ST A to MCA and STA to ACA) bypass and indirect encephalo-duroarterio-myo-synangiosis and encephalo-galeo-arteriosynangiosis for MCA and ACA territories, respectively. Cases included eight pediatric (as young as 5 years of age) and eight adult patients. There was only one reported minor infarct and no other complications in the 26 procedures. This alone is a technical feat, and the results are surely compelling. 
The authors attribute their superior results to the comprehensive revascularization strategy, where more conservative operations, especially the lack of STA-ACA direct bypass, would leave patients vulnerable to immediate perioperative risks in that territory. However, that is not necessarily a consistent proposition. Reported complications of surgery for moyamoya disease in the published literature and in anecdotal experience are not always related to the ACA territory. And there were apparently no complications related to staging second hemisphere revascularization in cases where the authors felt it was needed. The authors tell us that they managed 35 hemispheres in 22 patients with moyamoya disease during the same period, hence the majority of cases received the extensive procedure. And they state that they did not carry out planned ACA direct anastomosis in some cases for technical reasons. But the authors do not report results of intention to treat decision, where such strategy was attempted and could not be carried out (in up to 6 patients and 9 hemispheres), and what was the outcome of those cases.

The authors attempt to articulate specific indications for this aggressive procedure, but they apparently ultimately performed it on the majority of moyamoya cases in their series, and they admit that they attempted it in even more patients. Hence we are left at loss as to when such aggressive strategy would NOT be justified... Nevertheless, the authors do demonstrate apparent feasibility and safety of this very elegant operation in their patients and in their hands. We would caution against generalization to cases of moyamoya syndrome in other experience, including adult patients outside Japan, where basal occlusive vasculopathy may have different etiologies, and disease course, and where scalp vessel may not allow direct ACA anastomosis in most instances. And we must await confirmation of similar feasibility and safety when this approach is attempted on similar patients by other surgeons.

Issam A. AwAD, M.D., M.Sc., F.A.C.S., M.A. (Hon.) Evanston Northwestern Healthcare Department of Neurological Surgery Northwestern University Feinberg School of Medicine Evanston, Illinois, U.S.A.

This interesting paper raises several important points, among them that improving flow in one area may alter the hemodynamics enough to cause more severe ischemia in an adjacent area with previous borderline flow. The anterior cerebral artery territory tends to be neglected in ordinary ischemic situations but is obviously more at risk in moyamoya disease where the vascular changes are so widespread. The authors have obviously a lot of experience with this condition, and have suggested useful additions to the usual bypass procedures.

Another important point, raised in their discussion, is the significance of frontal lobe blood flow and function in children versus adults. The developing brain may well be more vulnerable to ischemia at this stage, particularly from the point of view of future intellectual performance. However, it is equally interesting that two of the patients with measured improvement in IQ were adults (Table 1); frontal lobe impairment to the extent seen here is probably important at any age.

Nicholas W. C. Dorsch, M.D., F.R.C.S., F.R.A.C.S. Department of Neurosurgery Westmead Hospital Sydney, Australia

The conventional and routine surgical technique for moyamoya disease covers mostly the middle cerebral artery territory by STA-MCA direct bypass and encephalosynangiosis using the temporal muscle. In other words, revascularization to the anterior cerebral artery territory is not always satisfactory using the conventional surgical technique including direct and indirect bypass surgery.

In this paper, Ishikawa et al. have reported an excellent technique to overcome these drawbacks. They have aggressively performed STA-ACA direct bypass for patients with moyamoya disease and its outcome is excellent. This is a quite unique and enthusiastic challenge.

However, this technique is quite intricate and not always accepted as a general option for this disease. There are some questions raised by this paper. Among them, the indication of this technique is quite controversial. It is obvious that this surgery takes more time and seems to be more invasive compared to the other conventional techniques. In my personal series, transient ischemic attack due to ischemia of the anterior cerebral artery territory is not always permanent after successful revascularization surgery even using the conventional techniques. It seems, therefore, the indication of this complicated surgery seems to be narrower than described in this paper.

I believe that this technique is theoretically acceptable for very limited cases of moyamoya disease. However, it has to be emphasized that this technique requires a superb background in fine microsurgical vascular anastomosis technique and considerable experience in the treatment of moyamoya disease.

Kiyohiro HoukIN, M.D. Department of Neurosurgery Sapporo Medical University Sapporo, Hokkaido, Japan 